\title{
O ACESSO ÀS INFORMAÇÕES SOBRE DIAGNÓSTICO E INTERNAÇÃO À PESSOA COM TRANSTORNO MENTAL ${ }^{1}$
}

O objetivo deste trabalho foi identificar o que os profissionais de saúde que trabalham em um hospital psiquiátrico pensam sobre o acesso às informações, sobre a doença e a internação. Foi realizada uma pesquisa descritiva, com abordagem qualitativa, em um hospital psiquiátrico. Participaram do estudo 33 profissionais que vivenciam o processo de hospitalização e cuidados diretos ao paciente. Os dados foram obtidos mediante entrevista semiestruturada e observação participante e apresentados nas categorias temáticas: "O paciente e as informações prestadas: normas e rotinas do hospital"; "O papel dos profissionais de saúde no oferecimento de informações aos pacientes"; "A insuficiência das informações e suas causas". Conclui-se que o acesso às informações pelas pessoas com transtornos mentais sobre seu transtorno e tratamento é limitado, o que evidencia a necessidade de promoção de ações de conscientização dos profissionais de saúde a respeito de seu papel na prestação de informações, para que assim o direito à informação seja garantido efetivamente.

Descritores: Direito à Saúde; Direito dos Pacientes; Acesso à Informação; Saúde Mental; Hospitalização; Pessoas Mentalmente Doentes.

\footnotetext{
${ }^{1}$ Artigo extraído da dissertação de mestrado "O Direito Humano à Saúde Mental: compreensão dos profissionais da área" apresentada à Escola de Enfermagem de Ribeirão Preto, Universidade de São Paulo, SP, Brasil. Apoio financeiro do Conselho Nacional de Desenvolvimento Científico e Tecnológico (CNPq). Este trabalho recebeu menção honrosa no XII Encontro de Saúde Mental 2012, Ribeirão Preto, SP, Brasil.

2 Doutoranda, Escola de Enfermagem de Ribeirão Preto, Universidade de São Paulo, Centro Colaborador da OPAS/OMS para o Desenvolvimento da Pesquisa em Enfermagem, Ribeirão Preto, SP, Brasil.

${ }^{3} \mathrm{PhD}$, Professor Associado, Escola de Enfermagem de Ribeirão Preto, Universidade de São Paulo, Centro Colaborador da OPAS/OMS para o Desenvolvimento da Pesquisa em Enfermagem, Ribeirão Preto, SP, Brasil.
}

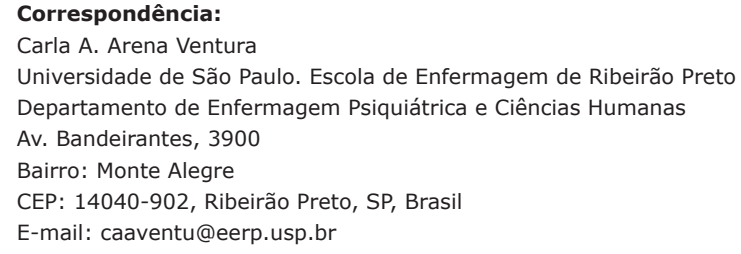




\section{ACCESS TO INFORMATION ON DIAGNOSING AND HOSPITALIZING AN INDIVIDUAL}

\section{WITH MENTAL ILLNESS}

The aim of this study was to identify what health care professionals working in psychiatric hospitals think about access to information concerning the disease and hospitalization. Descriptive research using a qualitative approach was conducted in a psychiatric hospital. A total of 33 health care professionals with experience of hospitalizing and directly caring for patients participated in the study. The data were obtained using a semi-structured interview and participant observation and are presented in thematic categories: "The patient and the information provided: hospital norms and routines"; "The role of health care professionals in providing patients with information"; "Lack of information and its causes". It was concluded that the access those with mental disorders have to information about their disorder and treatment is limited, showing the need to encourage awarenessraising activities in health care professionals about their role in providing information, to ensure the right to information is effectively guaranteed.

Descriptors: Right to Health; Patient Rights; Access to Information; Mental Health; Hospitalization; Mentally Ill Persons.

\section{EL ACCESO A LAS INFORMACIONES SOBRE DIAGNÓSTICO E INTERNAMIENTO DE LA PERSONA CON TRASTORNO MENTAL}

El objetivo de este trabajo fue identificar, lo que los profesionales de la salud que trabajan en un hospital psiquiátrico piensan sobre el acceso a las informaciones, sobre la enfermedad y el internamiento. Fue realizada una investigación descriptiva, con un abordaje cualitativo, en un hospital psiquiátrico. Participaron en el estudio 33 profesionales que se ocupaban del proceso de la hospitalización y cuidados directos al paciente. Los datos fueron obtenidos mediante entrevista semi-estructurada y observación activa y presentados en las categorías temáticas: "El paciente y las informaciones prestadas: normas y rutinas del hospital"; "El papel de los profesionales de la salud en el ofrecimiento de información a los pacientes"; "la insuficiencia de las informaciones y sus causas". Se concluye que el acceso a la información de las personas con trastornos mentales sobre su trastorno y tratamiento es limitado, lo que evidencia la necesidad de promover acciones de concienciación de los profesionales de la salud al respecto de su papel en la prestación de informaciones, para que así el derecho a la información sea garantizado efectivamente.

Descriptores: Derecho a la Salud; Derechos del Paciente; Acceso a la Información; Salud Mental; Hospitalización; Pessoas Mentalmente Doentes. 


\section{Introdução}

Receber o maior número de informações a respeito de sua doença e tratamento é um direito garantido às pessoas com transtornos mentais em diversos instrumentos legais (Lei Federal 10.216/01; Portaria do Ministério da Saúde no 1286/1993; Lei do estado de São Paulo 10.241/1999; Resolução do Conselho Federal de Medicina 1.407/1994; Resolução do Conselho Federal de Medicina 1.598/2000, Carta dos Direitos dos Usuários da Saúde do Brasil).

A Lei 10.241 de 1999 do estado de São Paulo $^{(1)}$ dispõe em seu artigo $2^{\circ}$, inciso VI, que são direitos dos usuários dos serviços de saúde do estado de São Paulo receber informações claras, objetivas e compreensíveis sobre: hipóteses diagnósticas e diagnósticos realizados; exames solicitados; ações terapêuticas; riscos e benefícios das medidas diagnósticas e tratamentos propostos; duração prevista do tratamento proposto; exames e condutas a que será submetido; a finalidade dos materiais coletados para exame; alternativas de diagnósticos e terapêuticas existentes no serviço de atendimento ou em outros serviços.

O segundo princípio da Carta dos Direitos dos Usuários da Saúde do Brasil assegura ao cidadão o tratamento adequado e efetivo para seu problema, visando à melhoria da qualidade dos serviços prestados, tendo garantidos informações sobre o seu estado de saúde, extensivas aos seus familiares e/ou acompanhantes, de maneira clara, objetiva, respeitosa, compreensível e adaptada à condição cultural, respeitados os limites éticos por parte da equipe de saúde sobre hipóteses diagnósticas e diagnósticos confirmados, dentre outras informações ${ }^{(2)}$.

Assim, o direito à informação sobre o diagnóstico é um meio que o paciente dispõe para tomar conhecimento e ter poder de determinação acerca do que está vivendo ${ }^{(3-4)}$. A falta de informação permite ações abusivas, sendo, portanto, necessário que o paciente conheça o seu diagnóstico e também os seus direitos para que possa reivindicá-los e exigir um melhor tratamento, possibilitando o exercício efetivo do direito.

Nesse sentido, este artigo buscou identificar o que os profissionais de saúde que trabalham em um hospital psiquiátrico pensam sobre o acesso às informações sobre a doença e a internação.

\section{Método}

Trata-se de pesquisa descritiva com abordagem de dados qualitativa. A abordagem de dados qualitativa é considerada apropriada quando o fenômeno em estudo é complexo, de natureza social e que não tende à quantificação. Busca interpretar os sentidos e os significados - de natureza psicológica e socioantropológica - atribuídos e trazidos por indivíduos acerca dos múltiplos fenômenos pertinentes ao campo no qual ocorre o processo saúde-doença ${ }^{(5)}$.

Os dados foram coletados mediante entrevista em profundidade semiaberta e gravada em meio digital. Foi escolhida essa técnica por explorar um assunto a partir da busca de informações, percepções e experiências dos informantes, que o entrevistador analisa e apresenta de forma estruturada $^{(6)}$. É extremamente útil para estudos do tipo descritivo, que buscam mapear uma situação ou campo de análise, descrever e focar determinado contexto. O modelo de entrevista semiaberta tem origem em um roteiro-guia de questões que dão cobertura ao interesse de pesquisa. Assim, o roteiro para a entrevista dos profissionais foi elaborado com base nos objetivos do estudo, focando as questões: Durante a hospitalização quais informações você fornece ao paciente? Você acha as informações suficientes?

Para serem incluídos no estudo, os sujeitos deveriam participar do processo de hospitalização e realizar cuidados diretos ao paciente internado, bem como concordarem em participar da pesquisa por meio da assinatura do Termo de Consentimento Livre e Esclarecido. A escolha dos profissionais como sujeitos do estudo se deve ao seu importante papel na garantia dos direitos dos pacientes. Nessa perspectiva, para que se assegure o respeito à lei, e para que seja prestada uma assistência considerando a humanidade de quem é cuidado, faz-se necessário que os profissionais envolvidos estejam permanentemente atentos aos direitos de seus pacientes enquanto usuários do sistema de saúde.

Dos 92 profissionais de saúde que trabalhavam no local de estudo, 33 aceitaram participar, sendo: cinco médicos, sete enfermeiros, 14 auxiliares de enfermagem, três técnicos de enfermagem, dois assistentes sociais, um psicólogo, um terapeuta ocupacional. A maioria era do sexo 
feminino $(63,63 \%)$, casados $(51,51 \%)$, auxiliares de enfermagem $(42,43 \%)$, com tempo de trabalho na profissão de 1 mês a 5 anos (63,64\%), com tempo de trabalho no local de estudo a de 1 mês a 5 anos $(69,7 \%)$.

Além da entrevista, a observação participante complementa em triangulação a metodologia. A observação participante é muito utilizada na abordagem qualitativa, uma vez que proporciona comprovar ou não os dados obtidos na entrevista, pois nem sempre os relatos dos sujeitos são compatíveis com o que é demonstrado em seus comportamentos. "Na observação participante, tem-se a oportunidade de unir o objeto ao seu contexto, contrapondo-se ao princípio de isolamento no qual fomos formados"(7). Nessa fase, foram seguidas as três etapas essenciais da observação participante ${ }^{(8)}: 1^{\text {a }}$ ) Aproximação do pesquisador com o grupo em estudo; $2^{\mathrm{a}}$ ) Obtenção da visão de conjunto deste grupo. Essa etapa foi realizada com o estudo de documentos oficiais; reconstituição da história do grupo e do local; observação da vida cotidiana; levantamento de pessoas-chave e entrevistas não direcionadas com as pessoas que poderiam ajudar na compreensão da realidade; $3^{a}$ ) Sistematização e organização dos dados ${ }^{(7)}$.

Essa fase da pesquisa foi desenvolvida durante um mês, entre os dias 06/12/2010 a 07/01/2011 e era realizada 4 vezes por semana durante aproximadamente 3 horas para cada visita. Procurou-se alternar as visitas entre os turnos da manhã, tarde e noite, entre diferentes dias da semana e entre todas as alas do hospital estudado. A observação possibilitou a imersão na realidade do universo estudado e maior aproximação com os sujeitos do estudo. O instrumento utilizado para o registro do trabalho de observação foi o diário de campo.

O estudo foi aprovado pelo Comitê de Ética da Escola de Enfermagem de Ribeirão Preto, sob o $\mathrm{n}^{\mathrm{o}} 1056 / 2009$ e todos os preceitos éticos foram salvaguardados atendendo à Resolução 196/96, do Conselho Nacional de Saúde. Os sujeitos receberam as informações sobre os objetivos do estudo sendo-lhes garantido o sigilo e anonimato. Para tanto, foram identificados com uma sigla e o respectivo número da entrevista. As siglas utilizadas foram: Enfermeiro-E; Auxiliar de enfermagem-AE; Técnico de enfermagem-TE;
Médico-M; Assistente Social-AS; Psicóloga-P; Terapeuta Ocupacional-TO.

Os dados foram coletados no período de dezembro de 2010 a janeiro de 2011, em um hospital psiquiátrico, situado no interior do Estado de São Paulo.

Para a análise dos dados foi aplicada a técnica de análise de conteúdo por categorias temáti$\operatorname{cas}^{(9)}$. As categorias foram construídas conforme os temas que emergiam do texto. A exploração do material foi composta por três fases ${ }^{(9)}$ : a pré-análise, em que os dados foram transcritos e organizados; na fase de exploração do material, os dados foram organizados por categorias e subcategorias temáticas, revisados repetidas vezes e codificados continuamente. Para a codificação, realizou-se a leitura exaustiva e repetida do conteúdo das entrevistas e dos recortes que representam significado para responder aos objetivos do estudo. Por fim, foi realizada a terceira fase, de tratamento e interpretação dos resultados que foram analisados, com subsídio da literatura sobre o objeto de estudo, e também dos dados da observação, culminando nas considerações finais.

Após a sistematização dos dados, foram identificadas as categorias temáticas: "O paciente e as informações prestadas: normas e rotinas do hospital"; "O papel dos profissionais de saúde no oferecimento de informações aos pacientes"; "A insuficiência das informações e suas causas".

\section{Resultados e discussão}

Quanto às informações fornecidas ao paciente, a maioria dos entrevistados da equipe de enfermagem disseram que fornecem apenas informações sobre as normas e rotinas do hospital.

Normas e rotinas da instituição (AE24).

a gente fornece mais informação de rotina do hospital (E12).

Quando eles internam isso aí já vêm lá da sala da enfermeira, a família já assina um termo já sabendo tudo que pode e que não pode fazer aqui dentro, o paciente já entra aqui dentro orientado sabendo o que ele tem ou não tem direito, a gente geralmente não passa muita informação (AE31).

as informações que a gente fornece é que ele tá num hospital psiquiátrico que aqui tem algumas restrições, é diferente de outros hospitais, o hospital clínico no caso, E que, por exemplo, tem coisas que eles não vão poder fazer e têm coisas que eles vão 
poder tá fazendo, igual por exemplo, a terapia é fundamental no tratamento dele, os horários de medicações, horário de janta, tudo isso a gente explica pra eles no momento da internação (AE21).

Os profissionais de saúde, especialmente os membros da equipe de enfermagem, focam, dentre as informações necessárias aos pacientes, as normas e as rotinas do hospital. Acreditam que essas sejam as informações mais úteis ao paciente, pois, afinal, devem cumpri-las corretamente para manter a organização no processo de cuidado.

O modelo organizacional do hospital apresenta características da burocracia tida como tipo ideal de estrutura organizacional ${ }^{(10)}$, baseando-se nos princípios de disciplina, especialização das funções, hierarquia, autoridade legal e formalização. Esse modelo de organização é um dos mais eficientes, uma vez que as ações são definidas por normas, o que leva a um comportamento disciplinado das pessoas.

Ressalta-se que a formalização dos procedimentos por meio de regras é bastante importante no ambiente hospitalar, sendo necessária para a manutenção da ordem e para que o hospital funcione. Porém, o excesso de burocracia causa uma disfunção e pode levar ao engessamento da organização caracterizada pelo apego total às normas e rotinas ${ }^{(11)}$.

Dessa forma, algumas disfunções da burocracia também foram identificadas no local de estudo: internalização das regras e exagerado apego aos regulamentos; excesso de formalismo e de papelório; resistência a mudanças; despersonalização do relacionamento; categorização como base do processo decisorial; superconformidade às rotinas e procedimentos; exibição de sinais de autoridade; dificuldade no atendimento a clientes e conflitos com o público ${ }^{(12)}$.

Verifica-se, que os profissionais se apegam às normas estabelecidas e às suas funções dentro da instituição de tal forma que acabam por burocratizar também o cuidado a pessoa com transtorno mental.

Meu papel, é muito burocrático, a gente fica muito nos papéis, mais nos papéis do que deveria, eu tenho um contato muito direto com os pacientes, eu gosto de entrar e conversar com o paciente, ter um diálogo sempre, mas a gente fica muito restrito aos papéis, como enfermeira chefe, muito papel e pouca assistência se for ver, mas sempre que eu posso tenho contato direto com o paciente (E26).

Observa-se que os profissionais possuem clareza com relação às suas funções, estabelecidas por normas internas, no processo de cuidado. Do mesmo modo, essa superconformidade às rotinas e procedimentos os levam a enfrentar dificuldades para a realização de mudanças, visando o aperfeiçoamento do processo de trabalho e até mesmo o incremento de suas habilidades, uma vez que se apegam demais à realização das suas funções predeterminadas pelos regulamentos.

Essa disfunção fica evidente também quando se fala no papel dos profissionais de saúde no oferecimento de informações ao paciente. Segundo os entrevistados, as informações sobre o tratamento devem ser dadas apenas pelo médico. As falas demonstram algumas das disfunções citadas anteriormente como a exibição de autoridade:

Sobre medicação, efeito colateral, esse tipo de coisa, a gente pede pra conversar com o médico, por mais que a gente saiba, às vezes a gente fala alguma coisa, depois o médico fala você não deveria ter falado isso, você não deveria ter falado que essa medicação dá esse efeito colateral que dai o paciente não quer tomar, alguma coisa desse tipo, entendeu? então interfere um pouco, então a gente pede pra falar sempre com o médico sobre esse tipo de coisa (E12).

O médico é para os participantes o profissional mais importante da equipe:

É o hospital, o médico é o cabeça, é o que muda, desmuda, prescreve, sabe o que o paciente tem ou não tem, o paciente chega e fala assim, eu tomo defakene, pra que é? Mesmo que eu saiba, eu não falo, não; você vê com o médico, o médico te informa pra que que serve, então o médico é tudo aqui, é a cabeça do hospital, se não fosse o médico o hospital não estaria funcionando (AE31).

Segundo os sujeitos da pesquisa, ao enfermeiro cabe acolher e cuidar do paciente:

A função do enfermeiro é de acolher o paciente e a família, porque muitas vezes essa familia e esse paciente vem assim é... ele não conhece a instituição, ele não conhece o que vai, ele não sabe o que vai acontecer com ele aqui dentro, então a função do enfermeiro é de recepcionar e de acolher essa familia e esse paciente primeiramente. Então, a nossa primeira função aqui quando a gente recebe o paciente no momento da internação é de acolhimento (E1).

Eu considero o meu papel completamente necessário, porque, apesar dele passar primeiro com a entrevista com o médico, é, o médico pergunta por exemplo, como ele é em casa, aí a 
família relata, então pra isso ele dá uma medicação, pra outro sintoma é outra medicação, a nossa parte é mais humana, não que o médico não seja humano, mas a gente acolhe o paciente, explica pra ele, qual a importância da internação, abraça, na verdade humaniza mesmo aquele paciente, não tratando ele como alguém que ocupa um leito mas como uma pessoa. Então é, esse é o papel da enfermagem em si (E2).

Éfunção também do enfermeiro supervisionar os técnicos e auxiliares de enfermagem.

O meu papel é supervisionar o serviço dos auxiliares, tá lá dentro vendo se tá dando certo o fluxo, se tá tudo correndo bem, se os pacientes tão com alguma necessidade, se os funcionários tão com alguma necessidade, remanejar funcionário. Tudo que acontece lá dentro com paciente, funcionário, é da minha [responsabilidade] (E12).

Além das funções de acolher, cuidar e supervisionar, ao enfermeiro cabe também, como integrante da equipe de saúde: participar no planejamento, execução e avaliação da programação de saúde; participar na elaboração, execução e avaliação dos planos assistenciais de saúde; prescrever medicamentos previamente estabelecidos em programas de saúde pública e em rotina aprovada pela instituição de saúde; participar nos programas e nas atividades de assistência integral à saúde individual e de grupos específicos, particularmente daqueles prioritários e de alto risco; participar em programas e atividades de educação sanitária, visando à melhoria de saúde do indivíduo, da família e da população em geral; participar nos programas de treinamento e aprimoramento de pessoal de saúde, particularmente nos programas de educação continuada; participar nos programas de higiene e segurança do trabalho e de prevenção de acidentes e de doenças profissionais e do trabalho; participar na elaboração e na operacionalização do sistema de referência e contrarreferência do paciente nos diferentes níveis de atenção à saúde e participar no desenvolvimento de tecnologia apropriada à assistência de saúde ${ }^{(13-14)}$.

Os técnicos e auxiliares em enfermagem resumem seus papéis à rotina de cuidados ao paciente.

A gente administra medicação, é... os cuidados, o curativo, às vezes a gente conversa também, dá apoio psicológico, chega fazer de todas as partes. (risos) (TE5).

O meu papel é... tá acolhendo essa pessoa e dando o melhor de mim pra cuidar (AE22).
Eu acho que é um papel muito importante porque a gente, porquê? Porque o médico não tá sempre em contato com o paciente. Então é a gente que tá em contato direto, que passa tudo, que analisa, que vê tudo, qualquer coisa que o paciente sente e tem, as diferenças, é a gente que leva até o médico, então eu acho que é um papel muito importante que a gente tem que fazer (AE8).

O meu papel? Cuidado. Cuidado geral do paciente, cuidado total, cuidado com vestimenta, cuidado com higiene, cuidado... (AE17).

O Decreto $n^{\circ}$ 94.406/87 que regulamenta o exercício da enfermagem dispõe que ao técnico de enfermagem cabe assistir o enfermeiro: no planejamento, programação, orientação e supervisão das atividades de assistência de Enfermagem; na prestação de cuidados diretos de enfermagem a pacientes em estado grave; na prevenção e controle das doenças transmissíveis em geral em programas de vigilância epidemiológica; na prevenção e controle sistemático da infecção hospitalar e na prevenção e controle sistemático de danos físicos que possam ser causados a pacientes durante a assistência de saúde ${ }^{(13)}$.

Além disso, são funções do auxiliar de Enfermagem preparar o pacientes para consultas, exames e tratamentos; observar, reconhecer e descrever sinais e sintomas, no nível de sua qualificação; executar tratamentos especificamente prescritos, ou de rotina, e também outras atividades de enfermagem, como: ministrar medicamentos por via oral e parenteral; realizar controle hídrico e fazer curativos; aplicar oxigenoterapia, nebulização, enteroclisma, enema e calor ou frio; efetuar o controle de pacientes e de comunicantes em doenças transmissíveis; realizar testes e proceder à sua leitura, para subsídio de diagnóstico; colher material para exames laboratoriais; executar atividades de desinfecção e esterilização ${ }^{(13)}$.

Também compete ao auxiliar prestar cuidados de higiene e conforto ao paciente e zelar por sua segurança, incluindo: alimentá-lo ou auxiliá-lo a alimentar-se; zelar pela limpeza e ordem do material, de equipamentos e de dependência de unidades de saúde; integrar a equipe de saúde; participar de atividades de educação em saúde, até mesmo orientar os pacientes na pós-consulta, quanto ao cumprimento das prescrições de Enfermagem e médicas e executar os trabalhos de rotina vinculados à alta de 
pacientes. Essas atividades somente poderão ser exercidas sob supervisão, orientação e direção do enfermeiro ${ }^{(13)}$.

Portanto, mais que acolher e cuidar das pessoas com transtornos mentais, a equipe de enfermagem deve participar ativamente no processo de decisão sobre o paciente ao lado da equipe multidisciplinar formada por médicos, psicólogos, assistentes sociais e terapeuta ocupacional.

"Com a reforma psiquiátrica, o campo teórico-prático da saúde mental passou a ser ressignificado exigindo que o enfermeiro abdique de seu papel de vigilância e controle das clientelas do manicômio para tornar-se, enquanto membro da equipe interdisciplinar, elemento essencial para intervir no processo re-habilitatório do sujeito com transtorno mental. Nesta conjuntura, um dos principais papéis do enfermeiro está relacionado ao vínculo, ao acolhimento e às intervenções mais humanas e eficazes consoantes às necessidades da vida concreta dos pacientes e suas famílias, buscando sempre compreender as necessidades dessas pessoas e viabilizar sua inserção social e preservar a saúde mental do grupo familiar"(15).

Uma vez que a sua proximidade no dia a dia com o paciente no hospital é maior, os profissionais da equipe de enfermagem possuem inúmeras oportunidades de oferecer uma orientação mais próxima ao paciente quanto às informações sobre diagnóstico e medicamentos. Mesmo assim, demonstraram em suas falas que acreditam não estarem aptos a fornecerem estas informações.

A gente não é o profissional adequado a tá dando essas orientações (AE24).

Então, a gente fala só o que cabe a enfermagem, orientações básicas mesmo (AE13).

Nesse cenário, resultados de estudo que avaliou o grau de conhecimento sobre a terapêutica medicamentosa apontou "deficiência nas ações educativas aos pacientes por parte dos profissionais de saúde, particularmente o enfermeiro, já que é o profissional que tem mais oportunidades de orientar e ensinar os pacientes em relação aos outros profissionais da equipe, embora não se exclua o papel fundamental do médico e do farmacêutico nessa atividade ${ }^{(16)}$ ".

Ainda com relação à terapêutica medicamentosa, os profissionais entrevistados acreditam que o conhecimento sobre a medicação pode acarretar a não adesão ao tratamento,

Às vezes, a gente não pode ficar falando muito sobre a medicação porque senão o paciente não vai aceitar o tratamento, não aceita, não quer internar então se você falar a medicação que ele vai tomar pra que serve, é mais uma restrição que ele não vai querer (AE20).

Ao contrário da opinião da maioria dos respondentes, a revisão da literatura sobre o tema enfatiza que a desinformação e/ou a não compreensão das informações pelos pacientes e familiares é um fator extremamente negativo para as pessoas com transtornos mentais, podendo ser determinante da não adesão ao tratamento ${ }^{(16-20)}$. Essa percepção sobre a importância do recebimento de informações foi destacada por um dos participantes:

Qual que é o objetivo dele estar aqui, tento dar uma ideia pra ele de qual o problema que ele tá apresentando naquele momento, porque que ele tá sentindo aqueles sintomas, se eu tiver um diagnóstico, esclarecer o diagnóstico que ele tem. Isso é sempre importante você passar pro paciente pra ele não se sentir inseguro, pra ele não pensar ninguém me explica nada, eu não sei o que tá acontecendo comigo. Então, eu oriento sempre sobre as medicações, o que a gente tá passando de remédio, qual é a função de cada um, isso no começo do tratamento cria um vínculo muito forte, se você fizer isso com o paciente ele vai colaborar muito mais no tratamento, ele vai aceitar as medicações mais facilmente, ele vai confiar mais em voce. Se ele achar que o remédio não tá fazendo efeito ele vai te contar, ele vai ter uma confiança muito maior em você, e consequentemente você vai ter mais sucesso no tratamento, porque ai os dois caminhando juntos fica muito mais fácil (M30).

Apesar da intenção de "informar", as informações segundo a maioria dos participantes da pesquisa não são suficientes. Os sujeitos justificaram a "insuficiência das informações" com base em dois motivos: o primeiro seria a falta de tempo para uma atenção individualizada.

Eu penso que ainda não seria, precisaria mais coisas, mas aí entra uma série de coisas por exemplo, o tempo, o tempo é muito curto, eu acho que precisaria mais, mas como nós estamos vivendo uma condição atípica hoje, até de farmacologia, o pessoal medica muito mais do que dá atenção ao paciente (M6).

Em estudo realizado em uma instituição psiquiátrica fechada e de grande porte, sobre a comunicação dos membros da equipe com o paciente e sua família, os pesquisadores verificaram que os profissionais atribuem grande importância à comunicação com os 
familiares das pessoas com transtornos mentais; porém, revelam que essa comunicação é quase impossível, pela falta de tempo para desenvolvê-la ${ }^{(21)}$.

O segundo motivo para a insuficiência das informações seria a dificuldade de compreensão das pessoas com transtornos mentais.

Seriam suficientes de acordo com o quadro do paciente no momento da internação, depende do jeito que o paciente tá internando isso daí ele não vai nem perceber, na realidade não tem como passar essas informações pra ele, dependendo do jeito que ele estiver sendo internado (AE17).

Os entrevistados falam da dificuldade de compreensão dos pacientes no momento da internação; no entanto, as informações devem ser fornecidas durante todo o processo de hospitalização, após o período de surto; quando já se estabeleceu um vínculo entre paciente e profissional fica mais fácil de o paciente com transtorno mental compreender o seu diagnóstico e tratamento. $\mathrm{O}$ transtorno mental traz dificuldades de comunicação, cabe aos profissionais proporcionar um ambiente acolhedor e manter uma relação de confiança com o paciente para que ele compreenda melhor sua situação e colabore mais efetivamente com o tratamento ${ }^{(22)}$.

\section{Conclusão}

A Lei Federal 10.216 de 2001 afirma ser direito da pessoa com transtorno mental: receber o maior número de informações sobre sua doença e seu tratamento.

Esta pesquisa buscou, assim, identificar o que os profissionais de saúde pensam sobre o direito de o paciente receber informações sobre seu transtorno e seu tratamento, uma vez que esses sujeitos exercem importante papel na prestação de informações e garantia dos direitos dos pacientes.

A partir da análise dos dados obtidos, conclui-se que o acesso às informações pelas pessoas com transtornos mentais sobre seu transtorno e seu tratamento é limitado. Os participantes reconhecem no médico o único profissional apto a prestar tais informações, cabendo aos demais profissionais prestarem apenas informações sobre as normas e as rotinas do hospital. Embora essas informações sejam disponibilizadas, são consideradas insuficientes pela maioria dos sujeitos devido à falta de tempo para uma atenção individualizada e à dificuldade de compreensão das pessoas com transtornos mentais.

Sugerimos a promoção de ações de conscientização direcionadas aos profissionais de saúde sobre seu papel na prestação de informações, para que, assim, o direito à informação seja garantido efetivamente. E também a realização de pesquisas que redirecionem o foco para a identificação do que os próprios usuários e seus familiares pensam acerca do seu direito de receber informações a respeito de seu transtorno e seu tratamento.

\section{Referências}

1. Lei estadual n. 10.241 , de 17 de março de 1999 (BR). Dispõe sobre os direitos dos usuários dos serviços e das ações de saúde no Estado e dá outras providências. [acesso 2 fev 2011]. Disponível em: http://www.saude.sp.gov.br/resources/profissional/acesso_rapido/gtae/saude_ da_mulher/lei_10241_direi tos_usuarios.pdf

2. Carta dos direitos dos usuários da saúde. Brasília: Ministério da Saúde; 2006.

3. Chaves PL, Costa VT, Lunardi VL. A enfermagem frente a direitos de pacientes hospitalizados. Texto Contexto Enferm. 2005;14(1):38-43.

4. Leite RAF. Direito à informação em saúde: análise do conhecimento do paciente acerca de seus direitos [dissertação de mestrado]. Ribeirao Preto (SP): Escola de Enfermagem de Ribeirão Preto da Universidade de São Paulo; 2010. 118 p. 5. Botega NJ, Turato ER. Pesquisa no hospital geral: primeiros passos. In: Botega NJ. Prática psiquiátrica no Hospital Geral: interconsulta e emergência. Porto Alegre: Artmed; 2006.

6. Duarte JAM. Entrevista em profundidade. In: Duarte JAM. Métodos e técnicas de pesquisa em comunicação. São Paulo: Atlas; 2005.

7. Queiroz DT, Vall J, Souza AMA, Vieira NFC. Observação participante na pesquisa qualitativa: conceitos e aplicações na área da saúde. Rev Enferm UERJ. 2007;15(2):276-83.

8. Richardson RJ. Pesquisa social: métodos e técnicas. São Paulo: Atlas; 1999.

9. Bardin L. Análise de conteúdo. Lisboa: Edições 70; 1995.

10. Weber M. Os fundamentos da organização burocrática: uma construção do tipo ideal. In: 
Campos E. Sociologia da burocracia. Rio de Janeiro (RJ): Zahar; 1978.

11. Timeni S. Teoria da Burocracia Weberiana e a relação com a Saúde Pública do RN. [acesso 30 jun 2011]. Disponível em: http://www. administradores.com.br/informe-se/artigos/ teoria-da-burocracia-weberiana-e-a-relacao-com-a-saude-publica-do-rn/51758/.

12. Motta FCP. Teoria geral da administração. São Paulo: Pioneira; 1991.

13. Decreto n. 94.406, de 8 de junho de 1987 (BR). Regulamenta a Lei 7.498, de 25 de junho de 1986, que dispõe sobre o exercício da Enfermagem, e dá outras providências. [acesso 5 jun 2011]. Disponível em: http:/www2.camara.gov.br/legin/fed/decret/1980-1987/decreto-94406-8-junho-1987-444430-publicacaooriginal-1-pe.html

14. Bocchi SCM. Characterization of nurses daily activities at an university hospital. Rev. Latino-Am. Enfermagem. 1996;4(21):41-58.

15. Jorge MSB, Freitas CHA, Luz, PM, Cavaleiro LMM, Costa RF. Enfermagem na atenção sistemática de saúde à família de pessoas com transtorno mental: estudo bibliográfico. Rev RENE. 2008;9(1):129-36.

16. Vianna CO, Opitz SP, Miasso AI, Linhares JC, Cassiani, SHB. Segurança do paciente hospitalizado: avaliação do grau de conhecimento sobre terapêutica medicamentosa. Esc Anna Nery. 2004;8(2):235-42.

17. Silva T, Schenkel EP, Mengue SS. Nível de informação a respeito de medicamentos prescritos a pacientes ambulatoriais de hospital universitário. Cad Saúde Pública. 2000;16(2):449-55.

18. Santos JLF, Furegato ARF, Osinaga VLM. Condicionantes do conhecimento do diagnóstico médico em três serviços psiquiátricos. Rev Enferm UFRJ. 2010;18(1):80-5.

19. Miasso AI, Cassiani SHB. Administração de medicamentos: orientação final de enfermagem para a alta hospitalar. Rev Esc Enferm USP. 2005;39(2):136-44.

20. Carvalho VT. Erros na medicação e consequencias para profissionais de enfermagem e clientes: um estudo exploratório. Rev Latino-Am Enfermagem. 2002;10(4):523-9.

21. Waidman MAP, Koga M, Stefanelli MC. Utilização da comunicação na prática da equipe multiprofissional de uma instituição psiquiátrica. Cogitare Enferm. 1997;2(1):82-5.
22. Salles MM, Barros S. Reinternação em hospital psiquiátrico: a compreensão do processo saúde/doença na vivência do cotidiano. Rev Esc Enferm USP. 2007;41(1):73-81. 